(C) 2018 IEEE. Personal use of this material is permitted. Permission from IEEE must be obtained for all other uses, in any current or future media, including reprinting/republishing this material for advertising or promotional purposes, creating new collective works, for resale or redistribution to servers or lists, or reuse of any copyrighted component of this work in other work.

\title{
INTER-COMPARISON OF ATMOSPHERIC CORRECTION METHODS ON SENTINEL-2 IMAGES APPLIED TO CROPLANDS
}

\author{
I. Sola; J. Álvarez-Mozos; M. González-Audícana \\ Public University of Navarre. Department of Projects and Rural Engineering, Arrosadia Campus, 31006 \\ Pamplona, Spain. Correspondence: ion.sola@unavarra.es; Tel.: +34-948-168401
}

\begin{abstract}
Atmospheric correction of high resolution satellite scenery is a necessary preprocessing step for applications where bottom of atmosphere (BOA) reflectances are needed. The selection of the best atmospheric correction method to use on images acquired from new platforms, such as Sentinel-2, is essential to provide accurate BOA reflectances. In this work the performance of three atmospheric correction methods (SEN2COR, MAJA and 6S) applied to Sentinel-2 scenes are compared by evaluating the resultant spectral signatures of six crop types on a single date, and their NDVI time series along a complete year. Although SEN2COR introduced greater corrections, especially in the infrared bands, the results suggest a varying performance of the methods depending on the land cover and the atmospheric conditions. Further research, particularly incorporating ground truth data, is recommended to rigorously validate the different atmospheric methods.
\end{abstract}

Index Terms - atmospheric correction, Sentinel-2, NDVI, crop, time series

\section{INTRODUCTION}

The Sentinel-2 (S2) mission is a land monitoring constellation of two satellites providing high resolution optical imagery, with a global coverage of the Earth's land surface every 5 days. The European Space Agency (ESA) Open Hub provides level-1C products, i.e., top of atmosphere (TOA) reflectance. Nevertheless, level-2A products, that is, atmospherically corrected bottom of atmosphere (BOA) reflectances are required for a plethora of applications. With this aim, different atmospheric correction (AC) methods have been developed in the last years. Although recently ESA started providing also level-2A S2 products corrected by the SEN2COR processor from March 2017, the selection of the best $\mathrm{AC}$ method for $\mathrm{S} 2$ imagery is a hot topic, currently under debate in the Atmospheric Correction Inter-Comparison Exercise (ACIX) [1]. Related to this, the objective of this work is to provide a comparison of three different $\mathrm{AC}$ methods applied to croplands. The performance of each method on different crop types is assessed, and their robustness is evaluated too by analyzing a complete year.

\section{MATERIALS AND METHODS}

\subsection{Study area}

An area of $20 \times 20 \mathrm{~km}$ was selected on the southern part of Navarre region (Spain), due to the presence of agricultural areas and a low cloud coverage during the year. This area is characterized by a Mediterranean climate and the predominant land cover are rain-fed and irrigated croplands. 


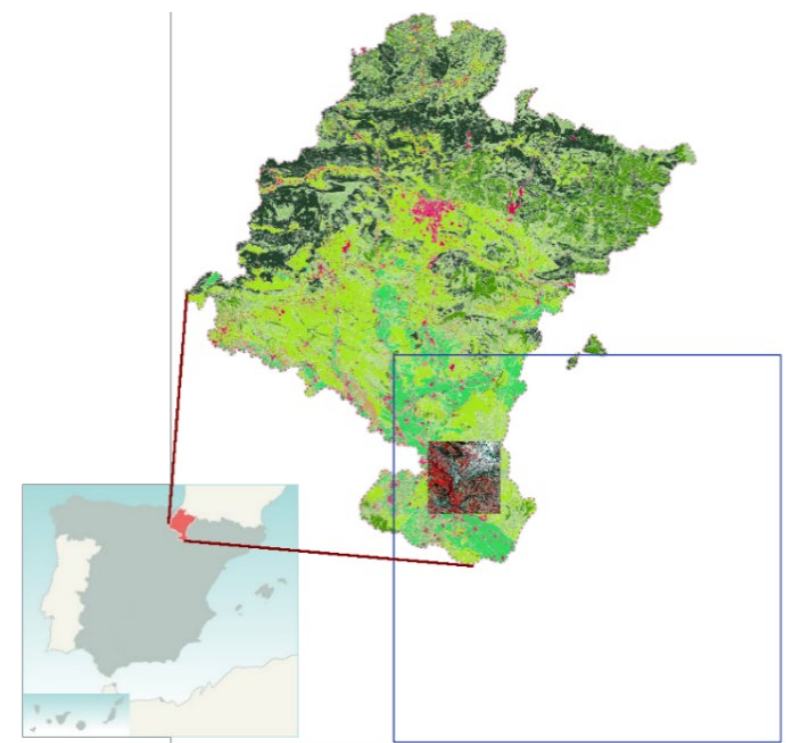

Figure 1. Study area located on the region of Navarre (Spain). Depicted in blue the frame of S2 T30TXM granule.

In the study area, 16 agricultural fields were selected, cultivated with 6 major crop types: wheat, barley, rice, corn, alfalfa and olive trees. The selection was based on the size of the fields (the bigger, the better) and the number of cloud-free S-2 images available for each field. A buffer of 10 meters was applied to avoid border pixel effects.

\subsection{AC methods}

S2 imagery were converted from TOA reflectances to BOA reflectances using three widely used AC methods. These methods were selected due to their popularity and high performance on different platforms.

\subsubsection{SEN2COR}

SEN2COR (2.3.1 version) is a processor developed by ESA to perform atmospheric correction, terrain and cirrus correction and scene classification applied to TOA Level-1C input data. The AC is performed using a set of Look-Up tables (LUT) generated with libRadtran [2]. These LUTs depend on Radiative Transfer Models (RTM) adjusted to the particular type of sensor, solar geometry, topography of the terrain and atmospheric parameters of the acquisition. The $\mathrm{AC}$ module is a porting and adaptation of ATCOR [3] into Python. Besides of BOA reflectances, the outputs are a Scene Classification (SCL) map along with quality indicators for cloud and snow probabilities, and aerosol optical thickness (AOT) and atmospheric water vapor (WV) maps.

\subsubsection{MAJA}

MAJA (MACCS ATCOR Joint Algorithm) [4] is a processor for cloud detection and $\mathrm{AC}$, specifically designed to process time series of high resolution optical images, acquired under quasi constant viewing angles, such as Landsat-8 or S-2. Since 2016, it is progressively including methods taken from German Aerospace Center's (DLR) ATCOR processor. The outputs are BOA reflectances with or without topographic correction (FRE and SRE, respectively). MAJA also provides a directory of masks including a cloud mask and a geophysical mask, and also maps of atmospheric parameters (WV, AOT). 

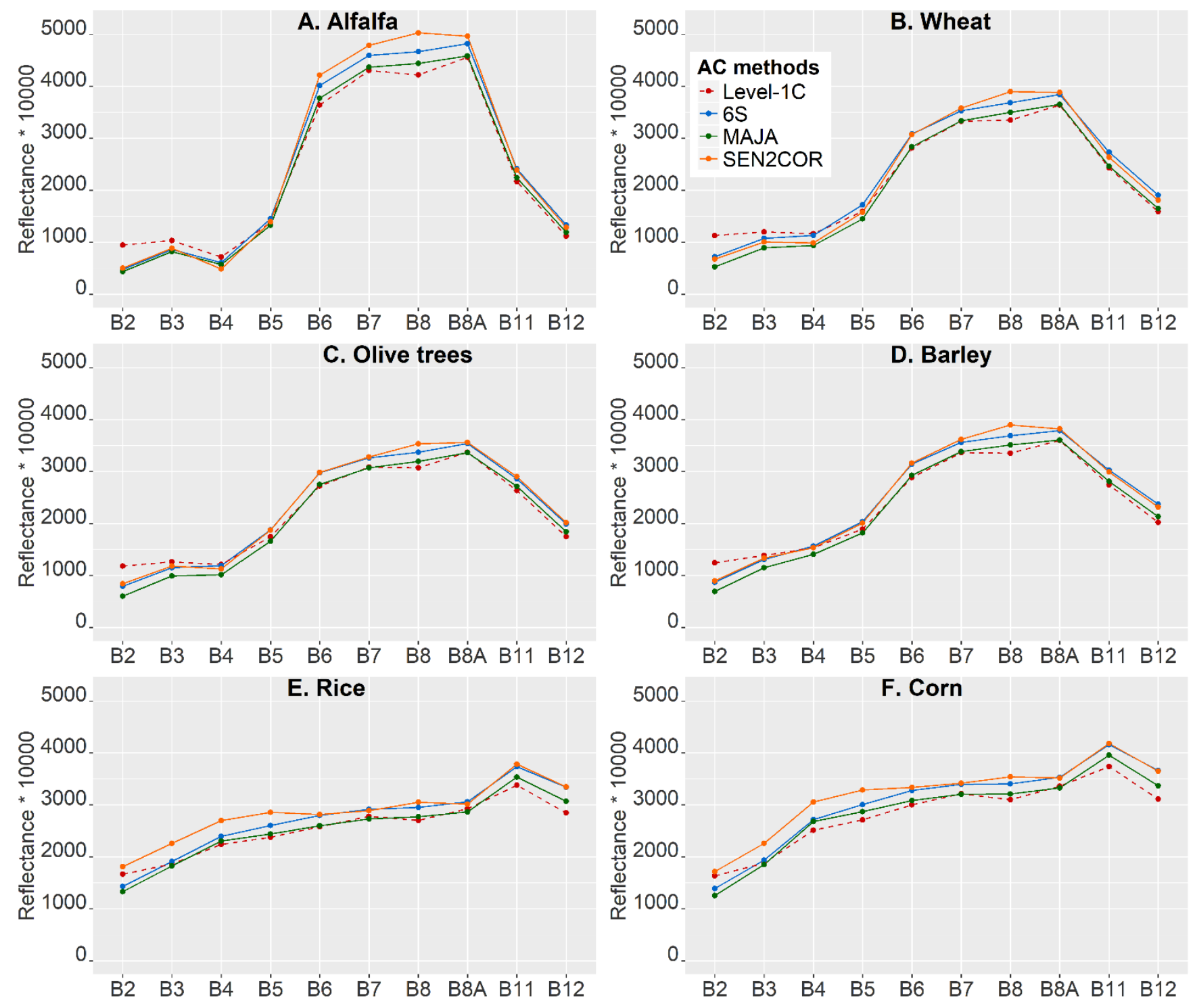

Figure 2. Spectral signatures of crop types obtained with the three different AC methods (6S, MAJA and SEN2COR), along with the uncorrected (1C) signature as a reference.

\subsection{3. $6 S$}

6S (Second Simulation of the Satellite Signal in the Solar Spectrum) is an open-source AC tool. In this work this method was applied using Py6S (an interface to the 6S atmospheric RTM through Python) [5, 6], on Google Earth Engine (GEE). 6S is generally used for the calculation of LUTs in the AC of MODIS. In contrast to the previous AC methods, the atmospheric parameters (WV, ozone and AOT) were not obtained from the image itself, but from GEE collections for the particular location, date and time of acquisition.

\subsection{Evaluation}

The performance of different AC methods was assessed through two different analyses. On the one hand, a single scene acquired during the vegetative period of most crops was selected (Table 1) and the spectral signatures of the crops obtained with the three AC methods were compared.

Table 1. Characteristics of the selected S2 image

\begin{tabular}{cccccc}
\hline Granule & Date & Time & $\begin{array}{c}\text { Cloud } \\
\text { coverage }\end{array}$ & $\begin{array}{c}\text { Solar } \\
\text { zenith }\end{array}$ & $\begin{array}{c}\text { Solar } \\
\text { azimuth }\end{array}$ \\
\hline \multirow{2}{*}{ T30TXM } & $\begin{array}{c}04 / 05 / \\
2016\end{array}$ & $10: 59$ & $0 \%$ & $28.1^{\circ}$ & $152.9^{\circ}$ \\
\hline
\end{tabular}

On the other hand, the time series of S2 imagery along one year were used to calculate the temporal curve of Normalized Difference Vegetation Index (NDVI), with the three AC methods. For that purpose, 23 S2 images (excluding images 
with cloud coverage higher than 50\%) of granule T30TXM of year 2016 were selected, and pixels covered by clouds or shadows were masked out. The availability of cloud-free images was from March to June, due to the bad weather conditions on these dates. On both analyses uncorrected Level-1C products were used a reference to interpret the results.

\section{RESULTS AND DISCUSSION}

\subsection{Spectral signatures of crop types}

The spectral signatures derived from BOA reflectances using the different $\mathrm{AC}$ methods are shown in Figure 2. When compared to Level-1C TOA reflectances, a clear decrease in blue reflectance is observed in most crop types. In the visible region, the higher the wavelength, the lower the reduction of reflectance after AC. This might be explained by the lower atmospheric scattering as wavelength increases. On the contrary, in the case of rice and corn the BOA reflectances obtained with SEN2COR were clearly higher than TOA reflectances, in contrast to other methods. It must be taken into account that rice and corn are summer crops not fully developed at the moment of image acquisition, so their reflectances in the visible region were higher than the other crops and lower in the near infrared,.

The values of red edge BOA reflectances (B5, B6 and B7) were similar to TOA reflectances in the case of cereals, olive trees and alfalfa, whereas they were higher in the case of rice and corn, especially with SEN2COR. In contrast, NIR bands (B8 and B8A) showed significantly higher values than TOA reflectances in the case of $6 \mathrm{~S}$, and to a greater extent with SEN2COR, while minor differences were observed between TOA reflectances and MAJA. Finally, the reflectance at short wave infrared bands (SWIR) was higher after AC, due to the strong atmospheric absorption in this region of the spectrum. The comparison of AC methods showed higher SWIR reflectance values with SEN2COR and 6S, and again MAJA introduced only slight differences to the original TOA reflectances.

\subsection{NDVI time series}

Figure 3 shows the NDVI time series for each crop type along year 2016. The number of image dates available from one crop type to another changed due to the varying cloud cover conditions on the different fields. The comparison of NDVI series obtained with different AC methods showed a clear increase of the index after $\mathrm{AC}$ in all the crop types and dates. This result was expected, due to the reduction of red reflectance and the increase of NIR reflectance produced by the atmospheric correction (see Figure 2).

Each crop type was characterized by a particular NDVI temporal curve, which showed significant differences depending on the AC method used. Winter cereals, such as barley and wheat, showed a similar pattern, characterized by a NDVI peak in May, with values up to 0.5-0.6. Similarly, summer crops like rice and corn had a NDVI peak, but later in time, around August. On the contrary, alfalfa showed a cyclical pattern as NDVI was strongly affected by the periodical cuts of the crop. As previous studies suggested [7] NDVI index tends to saturate in dense vegetation. This was the case with alfalfa in some dates here. In the case of MAJA, values of NDVI even higher than 1 were observed in some dates, and the behavior of the index seemed slightly more unstable than in SEN2COR and 6S, even if the cloudy scenes were discarded prior to the analysis.

In general, the NDVI curves along the growing season of crops showed apparent differences between AC methods, but these differences varied depending on the particular crop and no clear and consistent differences were observed. In the case of rice and corn MAJA and SEN2COR had higher NDVI values than $6 \mathrm{~S}$ during the whole year. By contrast, NDVI time series of cereals (i.e., wheat and barley) showed a more stable pattern with $6 \mathrm{~S}$, while MAJA and specially SEN2COR introduced significant changes from one date to another, due to the varying atmospheric conditions. Finally, olive trees showed a rather stable NDVI time series with values around 0.4 during the year, which might be a consequence of the understory vegetation between vines. The NDVI values obtained from TOA reflectances clearly increased after AC in most cases, showing a similar pattern during the year, with slight changes, except for MAJA, which introduced sharp changes in NDVI in a few cases. 


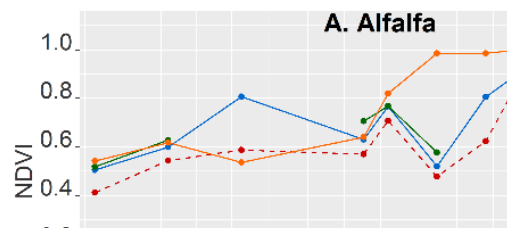

0.2

0.0

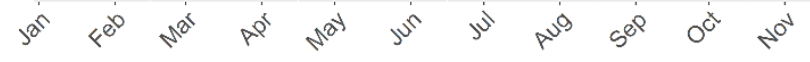

1.0

0.8

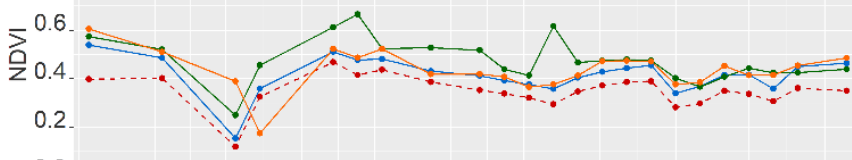

0.0
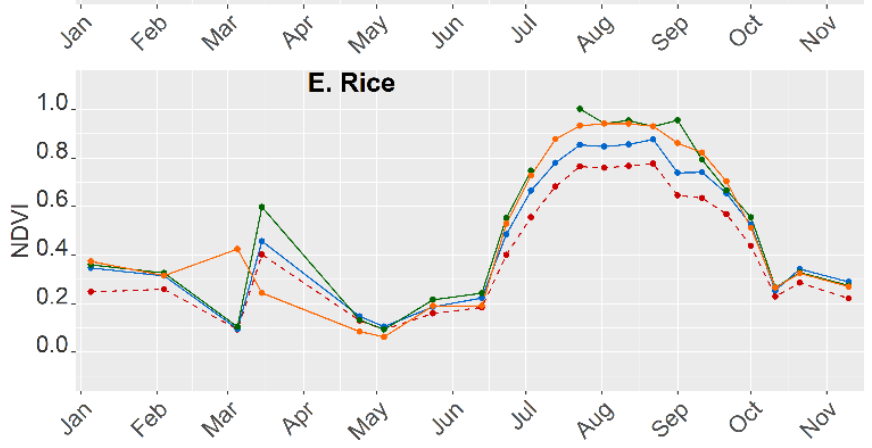

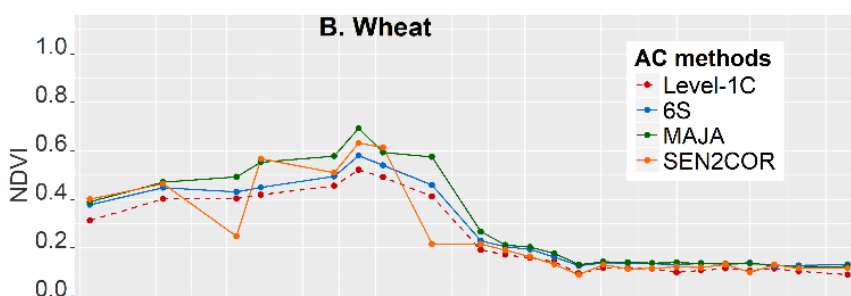

0.0

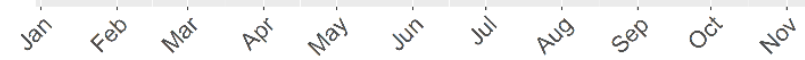

D. Barley

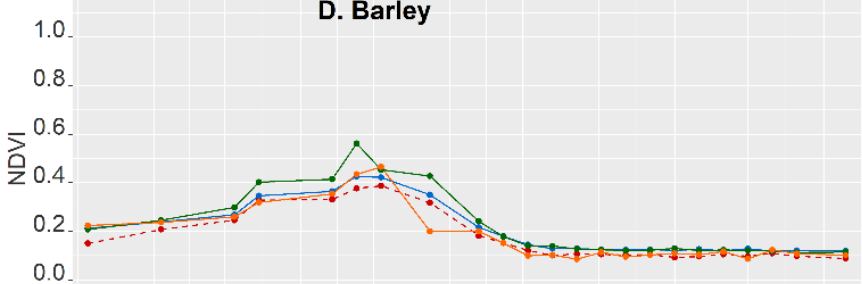

0.8

0.0

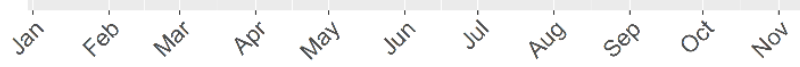

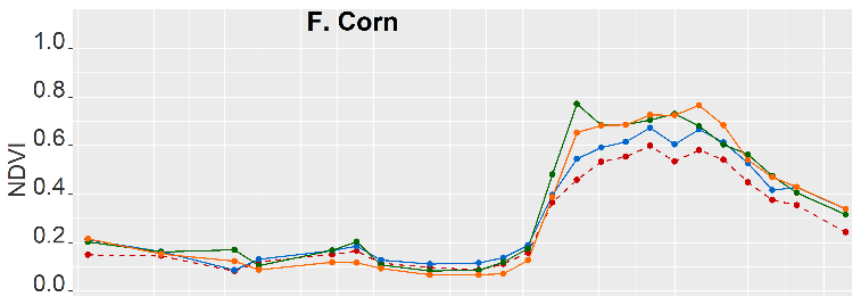

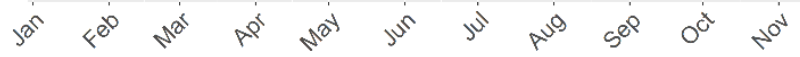

Figure 3. 2016 NDVI time series of each crop

\section{CONCLUSION}

The results obtained indicate that $\mathrm{AC}$ is a necessary preprocessing step particularly for multitemporal studies. Significant differences were observed between different methods, thus the selection of an adequate method seems a key factor in the preprocessing chain of S2 imagery. Furthermore, a clear increase in NDVI was observed after $\mathrm{AC}$, which proves the strong influence of atmospheric correction on S"-derived spectral indices.

Nevertheless, further research is required comparing S2-derived BOA reflectances with ground truth data, such as spectroradiometer measurements or validated BOA reflectances from alternative sources, e.g. MODIS. Moreover, the performance of associated additional products derived from S2 imagery, such as cloud masks and geophysical masks (detection of topographic shadows, cloud shadows, snow, water, etc.) is not assessed here, although it could also be a key factor to decide the best AC processor for $\mathrm{S} 2$ images.

\section{ACKNOWLEDGEMENTS}

This work is funded by the PyrenEOS EFA 048/15 project. This project has been $65 \%$ cofinanced by the European
Regional Development Fund (ERDF) through the Interreg V-A Spain-France-Andorra programme (POCTEFA 20142020).

\section{REFERENCES}

[1] Vermote, E.; Doxani, G.; Gascon, F.; Roger, J.-C. In ACIX: Atmospheric correction inter-comparison exercise, AGU Fall Meeting Abstracts, 2016.

[2] Mayer, B., Kylling, A. 2005. The libRadtran software package for radiative transfer calculations-description and examples of use. Atmospheric Chemistry and Physics, 5(7), 1855-1877.

[3] Richter, R., \& Schläpfer, D. 2005. Atmospheric/topographic correction for satellite imagery. DLR report DLR-IB, 565-01.

[4] Lonjou, V.; Desjardins, C.; Hagolle, O.; Petrucci, B.; Tremas, T.; Dejus, M.; Makarau, A.; Auer, S. In MACCS-ATCOR joint algorithm (MAJA), Proceedings of SPIE - The International Society for Optical Engineering, 2016.

[5] Murphy, S. Github repository of Sam Murphy. https://github.com/samsammurphy (accessed December 2017)

[6] Wilson, R. T., 2013, Py6S: A Python interface to the 6S radiative transfer model, Computers and Geosciences, 51, p166171. 
[7] Mutanga, O.; Skidmore, A. K. 2004. Narrow band vegetation indices overcome the saturation problem in biomass estimation. International Journal of Remote Sensing, 25(19), 3999-4014. 